\title{
Nano Technology in Medicine and Future Implications: A Mini Review
}

\section{Khursheed Ahmad Wani ${ }^{1 *}$ and Richa Kothari}

${ }^{1}$ Department of Environmental Science, ITM University, Gwalior, Madhya Pradesh, India

${ }^{2}$ Department of Chemistry, ITM University, Gwalior, Madhya Pradesh, India

\begin{abstract}
Nanoparticles hold great potential in the field of medicine. In this review we have discuuded some of the diseases that are treated by the use of nanotechnology. Nanotechnology has gained interest in recent years almost in every field with special emphasis on nanomedicine and nanobiology. Nanosystems with different compositions and biological properties have been extensively investigated for drug and gene delivery applications. However, it is important to understand the interactions of nanomaterials with the biological environment, targeting cell-surface receptors, drug release, multiple drug administration, stability of therapeutic agents under consideration.
\end{abstract}

\section{Keywords: Diseases; Nanomedicine; Target; Stability}

\section{Introduction}

The transformation of a molecule to nanoscale measure changes the properties of the material, for example, increment in surface region, strength of quantum impacts regularly connected with minute sizes, higher surface zone to volume proportion and so forth and fluctuates material's attractive, warm and electrical property. For instance, copper which is obscure at large scale ends up straightforward at nano scale. Thus the properties of gold at nanoscale makes change in dissolving point from $200^{\circ} \mathrm{C}$ to $1068^{\circ} \mathrm{C}$ and color changes from yellow to blue to violet alongside the adjustment in its synergist property [1].

However, ingested species may likewise impact the potential poisonous quality of the inhaled particles [2]. For nanoparticles the circumstance is diverse as their size opens the potential for intersection the different organic boundaries inside the body. From a positive perspective, particularly the possibility to cross the blood brain obstruction may open new routes for sedate conveyance into the cerebrum. Likewise, the nanosize additionally considers access into the cell and different cell compartments including the core. A large number of substances are as of now under scrutiny for the arrangement of nanoparticles for drug delivery, fluctuating from organic substances like egg whites, gelatine and phospholipids for liposomes, and more substances of a compound sort like different polymers and strong metal containing nanoparticles. Clearly, the potential association with tissues and cells, and the potential harmfulness, incredibly relies upon the genuine size of the nanoparticle detailing [3].

Numerous specialists append ethylene glycol particles to nanoparticles that convey remedial medications to growth tumors. The ethylene glycol atoms prevent white platelets from perceiving the nanoparticles as outside materials, enabling them to course in the circulatory system sufficiently long to connect to malignancy tumors. Currently, nanoparticles containing restorative medications covered with films from red platelets and it is demonstrated that these nanoparticles will flow in a mouse's circulatory system for just about two days, rather than the couple of hours as observed for nanoparticles utilizing ethylene glycol particles. Nanoparticles can be utilized as a part of focused medication conveyance at the site of infection to enhance the take-up of poorly soluble medications [4,5] the focusing of medications to a particular site, and medication bioavailability. A few drugs including paclitaxel [6,7], doxorubicin [8], 5-fluorouracil [9] and dexamethasone [10] have been effectively detailed utilizing nanomaterials by the studies. Polylactic/glycolic corrosive (PLGA) and polylactic corrosive (PLA) based nanoparticles have been detailed to exemplify dexamethasone, a glucocorticoid with an intracellular site of activity. Dexamethasone is a chemotherapeutic specialist that is hostile to anti-proliferative and anti-inflammatory effects. The medication binds to the cytoplasmic receptors and the consequent medication receptor complex is transported to the core bringing about the declaration of specific qualities that control cell multiplication [11]. The nanoparticles that discharge higher dosages of medication for delayed timeframe totally restrained expansion of vascular smooth muscle cells have been developed. Further, Scientists have built up a nanoparticle that uses a protein to connect to damage regions of veins [12]. This enables medications to be connected straightforwardly to the harmed portion of the artery. Experiments demonstrated that utilizing nanoparticles to focus on the conveyance of the coagulation busting drug may lessen conceivable side effects. The coagulation busting drug was connected to a group of nanoparticles that break separated in areas of tumultuous blood stream, similar to that found when a blood stream is limited by coagulation. Nanoparticles containing iron oxide that permits the nanoparticles to be coordinated, by a magnetic field, to stents [13]. This could enable medications to be conveyed straightforwardly to stents set in supply routes. Medications to treat glaucoma are being connected to nanodiamonds which are installed in contact focal points. Nanotechnology may have wider applications for treatment of eye disorders like drug delivery, study of pathomechanism of eye diseases, regeneration of the optic nerve, and counteracting neovascularization involved in some degenerative disorders. The medication particles are discharged from the nanodiamonds are in contact with tears, giving a more steady dosing than frequently happens utilizing eye drops [14].

Analysts are enhancing dental embeds by adding nanotubes to the surface of the embed material. They have shown to the capacity to stack the nanotubes with calming drugs that can be connected specifically to the region around the embed part [15]. Researchers have created nanoparticles that discharge insulin when glucose levels rise. The

*Corresponding author: Khursheed Ahmad Wani, Department of Environmenta Science, ITM University, Gwalior, Madhya Pradesh, India, Tel: 7513981200 E-mail: kuurshidevs@gmail.com

Received: March 26, 2018; Accepted: April 02, 2018; Published: April 09, 2018

Citation: Wani KA, Kothari R (2018) Nano Technology in Medicine and Future Implications: A Mini Review. J Nanomed Nanotechnol 9: 497. doi: 10.4172/21577439.1000497

Copyright: ( 2018 Wani KA, et al. This is an open-access article distributed under the terms of the Creative Commons Attribution License, which permits unrestricted use, distribution, and reproduction in any medium, provided the original author and source are credited. 
nanoparticles contain both insulin and a protein that break down in abnormal amounts of glucose [16].

Another strategy being created to discharge insulin utilizes a wipe like grid that contains insulin and also nanocapsules containing a chemical. At the point when the glucose level ascents the nanocapsules discharge hydrogen particles, which bind to the filaments making up the lattice [17]. The hydrogen particles make the filaments emphatically charged, repulsing each other and making openings in the framework through which insulin is discharged [18]. Researchers are creating nanoparticles that would delivery be able to drugs over the mind obstruction to handle neurologic clutters. A technique being created to handle immune system illnesses utilizes nanoparticles to convey antigens for a specific malady into the circulatory system. The antigens reset the invulnerable framework, preventing white platelets from assaulting sound cells. This strategy has been tried in the lab on mice with an ailment like different sclerosis with promising outcomes [19].

Skin creams those utilizations proteins got from immature microorganisms to avert maturing of the skin. These proteins are typified in liposome nanoparticles which converge with the films of skin cells to permit conveyance of the proteins. Experts have built up a nanoparticle that can sneak past bodily fluid covering surfaces, for example, lung tissue. This capacity to infiltrate the bodily fluid covering ought to give the ability to coat lung tissue with helpful medications [20].

\section{Liposome Nanoparticles}

Hydrophilic molecules can be encapsulated in the inner aqueous phase and hydrophobic molecules can be carried in the hydrophobic domains of the lipid bilayer. By simply mixing commercially available lipid molecules, physicochemical properties of liposomes can be accurately changed to control surface charge, functionality, and size that has an advantage over other carriers that need much more controlled synthesis steps with additional chemical alterations. DSPE (1,2-distearoylsn-glycero-3-phosphoethanolamine), HSPC (hydrogenated phosphatidylcholine from soybean lecithin), EggPG (egg yolk phosphatidylglycerol) and DSPC (1,2-distearoyl-glycero-3phosphocholine) have been used to set up vesicular formulations after approval by the FDA. PEG plays an important role in the formation of such lipids, which can be used to modify the surface of the resulting liposome. A novel discovery in the form of Doxil, a pegylated liposome has been clinically used to cure multiple types of cancer. Aphios Corp. manufactured nanosomes (small liposomes, $<100 \mathrm{~nm}$ ) having multiple drugs such as docetaxel, camptothecin, bryostatin-1 and vitamin D analog to cure multiple cancer types (Castor 2005) using a manufacturing technology based on a super-critical fluid process. Further, Novosom AG embedded with amphoteric liposomes to transport nucleic acids. The liposomal formulation may change zeta potential with changes in solution $\mathrm{pH}$ that may result in fusion during endocytosis uptake that permits nanocarriers into the cytoplasm for therapeutic load.

\section{Nanoparticle Technologies for Cancer Therapy}

It is very difficult to control drug release by using liposomal systems, however, if engineered by destabilizing lipids having amine head groups with vesicle membrane or including additives like morpholine in the lipid formulation [21-25]. LiPlasome Pharma manufactured non-targeted liposomes with lipids designed to be degraded by phospholipase A2 (PLA2), which is up-regulated in the tumor microenvironment [26-28] are transformed into anticancer drugs that will create local delivery of cytotoxic drugs in the tumor. Docetaxel is encapsulated into the liposome bi-layer and stabilized by albumin to prevent rapid drug leakage (ATI1123) that has been tested for efficacy studies in human xenograft mice models for prostate, pancreatic, and non-small-cell lung cancer (NSCL cancer) that resulted in partial tumor inhibition in $90 \%$ of the PC3 tumor xenograft model and enhanced efficacy in the pancreas model.

\section{Polymer-Drug Conjugates Nanoparticles}

Polymer- medicate conjugates are a standout amongst the most explored sorts of nanocarriers and are as of now in clinical trials as cutting edge as stage III. Polymer-medicate conjugates are framed through side-anchor joining of medications to polymer chains, enabling them to convey high measurements of chemotherapeutic medications. In spite of the fact that the physicochemical properties of various details are not uncovered, the measure of polymer-tranquilize conjugates is for the most part underneath $20 \mathrm{~nm}$. HPMA-doxorubicin (N-(2-hydroxypropyl) methacrylamide) copolymer (PK1) was the first engineered polymer-anticancer medication conjugate to enter clinical trials over 10 years prior and the clinical stage II trial for ladies with cutting edge bosom disease is as yet progressing [29]. Likewise, Prolindac (AP5346) is made out of a HPMA spine copolymer with platinum united to the side chains through a $\mathrm{pH}$-touchy chelator intended for sedate discharge in the tumor condition [30]. Preclinical information indicates prevalent efficacy of the polymer-medicate conjugates utilizing numerous malignancy models including a M5076 sarcoma platinum-safe tumor xenograft mice display, different colon xenograft models, L1210 leukemia, and 0157 hybridoma models [31]. Oxaliplatin medicate stacking was $\sim 10 \%(\mathrm{w} / \mathrm{w})$ utilizing a polymer chain of $25 \mathrm{kDa}$ and the medication discharge was moderate.

As of late, Access Pharmaceuticals Inc. detailed the consequences of the clinical stage II trial demonstrating that $66 \%$ of the patients with ovarian malignancy experienced significant ailment adjustment and restricted symptoms. Polyamino acids joined with drugs as an afterthought chains are another class of polymer-medicate conjugates that have shown high medication stacking and efficacy. On account of polyglutamate-glycine-campthotecin (CT-2106), degradable linkers have permitted sedate loadings going from $5 \%$ to half. Utilizing a glycine linker, medicate loadings were expanded triple over polyglutamate-campthotecin alone because of decreased steric obstacle. Be that as it may, a definition with a medication heap of $\sim 30 \%$ was chosen for clinical trials because of predominant soundness and efficacy in human tumor xenograft mice models [32]. In the interim, Xyotax, a comparative polymer-medicate conjugate (polyglutamatepaclitaxel), is in 22 clinical trials right now for numerous malignancy treatments including prostate growth, metastatic bosom tumor, neck disease, metastatic colorectal growth, and intermittent NSCL (Phase III). Paclitaxel is joined to polyglutamic corrosive $(30-40 \mathrm{kDa})$ to achieve a medication heap of $20-40 \%$ by weight [28]. The clinical information demonstrates a change in middle survival in Xyotax patients contrasted and the control gathering, despite the fact that there were no distinctions in the general survival. One benefit of the treatment was the decrease of numerous reactions including neurotoxicity [25]. By and large, polymer-medicate conjugates are viewed as basic nanocarrier frameworks, however tuning the ideal detailing may require broad improvement. For instance, little changes in the polymer-medicate conjugation efficiency may significantly adjust the pharmacokinetic parameters and tissue biodistribution. The subsequent definition could likewise be viewed as another substance element, confusing administrative endorsement. 
Citation: Wani KA, Kothari R (2018) Nano Technology in Medicine and Future Implications: A Mini Review. J Nanomed Nanotechnol 9: 497. doi: 10.4172/2157-7439.1000497

\section{Conclusion}

Nanoparticles are quickly turning into the focal point of most endeavors going for targets and site-particular medication conveyance. The focusing on capacity of nanoparticles relies upon specific factors, for example, molecule measure, surface charge, surface adjustment and hydrophobicity. Constrained information about the poisonous quality of nanoparticles is a noteworthy concern and absolutely merits more consideration. The most encouraging examination in nanoparticle generation is by means of utilizing supercritical liquids which are natural agreeable and free of poisonous solvents. Much research is right now being performed to conquer these obstacles which will build up nanoparticle-based medication conveyance as the highest quality level for site-particular therapeutics.

There also is an exciting possibility to defeat issues of medication protection in target cells and to encouraging development of medications to overcome obstructions, for example, those in the brain. The test, nonetheless, remains the exact portrayal of atomic targets and to guarantee that these particles are communicated just in the focused on organs to anticipate consequences for sound tissues. Besides, it is essential to comprehend the destiny of the medications once conveyed to the core and other delicate cells organelles. Besides, on the grounds that nanosystems increment productivity of medication conveyance, the measurements may require recalibration.

\section{References}

1. Tiwari JN, Tiwari RN, Kim KS (2012) Zero-dimensional, one-dimensional, twodimensional and three-dimensional nanostructured materials for advanced electrochemical energy devices. Prog Mater Sci 57: 724-803.

2. Chen C, Xing G, Wang J, Zhao Y, Li, et al. (2005) Multihydroxylated [Gd@ $\mathrm{C} 82(\mathrm{OH}) 22] \mathrm{n}$ nanoparticles: antineoplastic activity of high efficiency and low toxicity. Nano Lett 5: 2050-2057.

3. Ibrahim K, Saeed K, Khan I (2017) Nanoparticles: Properties, applications and toxicities. Arabian Journal of Chemistry.

4. Ould-Ouali L, Noppe M, Langlois X, Willems B, Te Riele P, et al. (2005) Selfassembling PEG-p(CL-co-TMC) copolymers for oral delivery of poorly watersoluble drugs: a case study with risperidone. J Control Release 102: 657-668.

5. Kipp JE (2004) The role of solid nanoparticle technology in the parenteral delivery of poorly water-soluble drugs. Int J Pharm 284: 109-122.

6. Fonseca C, Simoes S, Gaspar R (2002) Paclitaxel-loaded PLGA nanoparticles: preparation, physicochemical characterization and in vitro anti-tumoral activity. J Control Release. 83: 273-286.

7. Andresen TL, Davidsen J, Begtrup M, Mouritsen OG, Jorgensen K (2004) Enzymatic release of antitumor ether lipids by specific phospholipase A2 activation of liposome-forming prodrugs. J Med Chem 47: 1694-1703.

8. Koziara JM, Whisman TR, Tseng MT, Mumper RJ (2006) In-vivo efficacy of novel paclitaxel nanoparticles in paclitaxel-resistant human colorectal tumors. J Control Release 112: 312-319.

9. Yoo HS, Lee $\mathrm{KH}$, Oh JE, Park TG (2000) In vitro and in vivo anti-tumor activities of nanoparticles based on doxorubicin-PLGA conjugates. J Control Release 68: 419-431.

10. Bhadra D, Bhadra S, Jain S, Jain NK (2003) A PEGylated dendritic nanoparticulate carrier of fluorouracil. Int J Pharm 257: 111-124.
11. Matsumura $Y$ (2008) Poly (amino acid) micelle nanocarriers in preclinical and clinical studies. Adv Drug Deliv Rev 60: 899-914.

12. Campen MJ1, McDonald JD, Gigliotti AP, Seilkop SK, Reed MD, et al. (2003) Cardiovascular effects of inhaled diesel exhaust in spontaneously hypertensive rats. Cardiovasc Toxicol 3: 353-361.

13. Borm PJ, Kreyling W (2004) Toxicological hazards of inhaled nanoparticles - potential implications for drug delivery. J Nanosc Nanotechnol 4: 521-531.

14. Jain KK (2008) Nano-Ophthalmology. In: The Handbook of Nanomedicine. Humana Press.

15. Bhardwaj A, Bhardwaj A, Misuriya A, Maroli S, Manjula S, et al. (2013) Nanotechnology in dentistry: Present and future. J Int Oral Health 6: 121-126.

16. Yamaguchi $Y$, Igarashi $R$ (2006) Nanotechnology for therapy of type 2 diabetes Nihon Rinsho 64: 295-300.

17. Joshi HM, Bhumkar DR, Joshi K, Pokharkar V, Sastry M (2006) Gold nanoparticles as carriers for efficient transmucosal insulin delivery. Langmuir 22: 300-305

18. Panyam J, Labhasetwar V (2004) Sustained cytoplasmic delivery of drugs with intracellular receptors using biodegradable nanoparticles. Mol Pharm 1: 77-84.

19. https://www.sciencedaily.com/releases/2007/10/071002163854.htm

20. Cullis PR, Chonn A (1998) Recent advances in liposome technologies and their applications for systemic gene delivery. Adv Drug Deliv Rev 30: 73-83.

21. Guo X, MacKay JA, Szoka FC Jr (2003) Mechanism of pH-triggered collapse of phosphatidylethanolamine liposomes stabilized by an ortho ester polyethyleneglycol lipid. Biophys J 84: 1784-1795.

22. Kocer A (2007) A remote controlled valve in liposomes for triggered liposoma release. J Liposome Res 17: 219-225.

23. Sudimack JJ, Guo W, Tjarks W, Lee RJ (2002) A novel pH-sensitive liposome formulation containing oleyl alcohol. Biochim Biophys Acta 1564: 31-37.

24. Vial F, Rabhi S, Tribet C (2005) Association of octyl-modified poly(acrylic acid) onto unilamellar vesicles of lipids and kinetics of vesicle disruption. Langmuir 21: 853-862

25. Andresen TL, Jensen SS, Kaasgaard T, Jorgensen K (2005) Triggered activation and release of liposomal prodrugs and drugs in cancer tissue by secretory phospholipase A2. Curr Drug Deliv 2: 353-362.

26. Jensen SS, Andresen TL, Davidsen J, Hoyrup P, Shnyder SD, et al. (2004) Secretory phospholipase A2 as a tumor-specific trigger for targeted delivery of a novel class of liposomal prodrug anticancer etherlipids. Mol Cancer Ther 3: 1451-1458.

27. Boddy AV, Plummer ER, Todd R, Sludden J, Griffin M, et al. (2005) A phase I and pharmacokinetic study of paclitaxel poliglumex (XYOTAX), investigating both 3-weekly and 2-weekly schedules. Clin Cancer Res 11: 7834-7840.

28. http://www.understandingnano.com/nanotechnology-drug-delivery.html

29. Jorgensen K, Davidsen J, Mouritsen OG (2002) Biophysical mechanisms of phospholipase A2 activation and their use in liposome-based drug delivery. FEBS Lett 531: 23-27.

30. Li C (2002) Poly(L-glutamic acid)-anticancer drug conjugates. Adv Drug Deliv Rev 54: 695-713.

31. Suri SS, Fenniri H, Singh B (2007) Nanotechnology-based drug delivery systems. J Occup Med Toxicol 2: 16.

32. Singer JW, Baker B, De Vries P, Kumar A, Shaffer S, et al. (2005) Poly-(L)glutamic acid-paclitaxel (CT-2103) [XYOTAX], a biodegradable polymeric drug conjugate: characterization, preclinical pharmacology, and preliminary clinical data. Adv Exp Med Biol 519: 81-99. 